\title{
Macrophage migration inhibitory factor promoter polymorphisms (-794 CATT5-8): Relationship with soluble MIF levels in coronary atherosclerotic disease subjects
}

Lu Qian ${ }^{1+}$, Xiao-Yan Wang ${ }^{2+}$, Saroj Thapa ${ }^{1}$, Lu-yuan Tao ${ }^{1}$, Shao-Ze Wu' ${ }^{1}$ Gao-Jiang Luo ${ }^{2}$, Lu-Ping Wang', Jiao-Ni Wang ${ }^{1}$, Jie Wang ${ }^{1}$, Ji Li ${ }^{1}$, Ji-Fei Tang ${ }^{1 *}$ and Kang-Ting Ji ${ }^{1 *}$ (D)

\begin{abstract}
Background: We analyzed the relationship of -794 CATT5-8 MIF polymorphisms with soluble MIF in Coronary Atherosclerotic Disease (CAD) patients.

Methods: A total of 256 patients selected, on which 186 normal-coronary and 70 Coronary artery disease subjects, were recruited in the study (Retrospectively registered). Genotyping of -794 CATT5-8 polymorphisms were performed by PCR and DNA sequencing. Serum MIF levels were measured using an ELISA kit. Patients were classified by coronary angiogram, and CAD based on Gensini's integral degree (angiographic scoring system).

Results: The allele frequency and genotype frequency of -794 CATT5-8 did not show any differences in normal-coronary subjects and CAD subjects. In CAD patients, serum MIF levels was lower in CATT (5) subjects than in CATT (7) subjects, while the genotype of -794 CATT5-8 did not show differences in serum MIF levels. In addition, we found a decrease in serum MIF levels in carriers of the (5/5) genotypes the -794 CATT5-8 MIF polymorphisms, although it was not significant. There was no relationship of CAD class and the allele frequency of -794 CATT5-8.
\end{abstract}

Conclusions: This study found no association between CAD class and -794 CATT5-8 MIF polymorphisms with soluble MIF levels in CAD Subjects.

Trial registration: NCT01750502 (November 2012, Retrospectively registered).

Keywords: Macrophage Migration Inhibitory Factor, Gene polymorphisms, Coronary Atherosclerotic Disease, Gensini's degree integral

\section{Background}

Coronary Atherosclerotic Disease (CAD) is characterized by atherosclerotic plaques in the vascular wall that results in vascular stenosis or plaque disruption with acute thrombotic occlusion. It occurs due to gradual cholesterol and fibrous tissue plaque in the wall of coronary artery over long period [1]. Various risk factors have been identified for CAD, such as smoking, hypercholesterolemia, hypertension, and diabetes [2].

\footnotetext{
* Correspondence: jefftang@medmail.com; ziguanger@163.com

${ }^{\dagger}$ Equal contributors

'Department of Cardiology, the Second Affiliated Hospital, Wenzhou Medical University, Wenzhou, Zhejiang 325000, China

Full list of author information is available at the end of the article
}

Evidence suggests that $\mathrm{CAD}$ is an inflammatory process with chronic inflammation of vessel wall infiltrated by circulating immune cells, such as monocytes and macrophages [3]. Macrophage migration inhibitory factor (MIF) is a homotrimer protein with a molecular weight of $37.5 \mathrm{kDa}$, which can promote the inflammation [4]. The first experimental studies that utilized pure recombinant MIF and neutralizing antibodies established that MIF played a critical role in the inflammatory cascade leading to endotoxic shock and death [5]. Soon thereafter, it was found that the macrophage, which had been considered historically to be the "target" of MIF action, was in fact a significant source of MIF production. In the case of the 
macrophage, MIF promotes TNF $\alpha$ production, which leads to further MIF release and a re-entrant activation pathway that is required for the optimal expression of TNF $\alpha$ and other pro-inflammatory mediators [6]. MIF is expressed in several cell types, including monocytes, macrophages, vascular smooth muscle cells (SMCs), and cardiomyocytes [6-8].

As described by Jie Wu et al. [9], the MIF gene maps to chromosome 21q22.33 in human (2119 bp). There are four polymorphisms that have been mainly reported in the human MIF gene, including three Single Nucleotide Polymorphisms (SNPs) at positions -173 (rs755622), +254 (rs2096525), and +656 (rs2070766) and a 794CATT5-8 microsatellite polymorphism. Loci rs2096525 and rs207 0766 are located in introns, whereas rs755622 and -794CATT5-8 are located in the promoter region of MIF [9] . Earlier studies have found that circulating MIF levels are elevated in ulcerative colitis (UC) [10], psoriasis [9] and tuberculosis (TB) [11]. Since these diseases are accompanied by persistent inflammation of varying degrees, it is possible that MIF may play a role in the development of these diseases.

Previous studies have reported that the plasma MIF level of CAD group was higher than non-CAD patients and the plasma MIF level was related to the stability of the plaque [12]. Also, we demonstrated a close association between the polymorphism of MIF on the -173 position and CAD [12]. The aim of this study was to investigate the relationship between -794 CATT5-8 MIF polymorphisms and soluble MIF levels in CAD patients.

\section{Methods}

\section{Subjects}

A total of 256 subjects were enrolled, including 186 without $\mathrm{CAD}$ and 70 with $\mathrm{CAD}$ subjects, in the period from 06/2012 to $12 / 2012$ in our inpatient department. All patients underwent coronary angiography (CAG) interpreted by one independent radiologist. Stenosis of the left main artery [13], Left Anterior Descending (LAD) branch, Right Coronary Artery (RCA), and other major branches were evaluated. CAD patients had the evidence of atherosclerosis (i.e., $\geq 50 \%$ luminal stenosis) in at least one coronary artery or major branch segment in their epicardial coronary tree. Patients in the control group had no luminal stenosis at CAG. Patients were excluded if they had acute inflammatory diseases, tumors, autoimmune disease and severe hepatic and renal dysfunction. All participants were of Han ethnicity living in Wenzhou, a southeastern coastal city of China. This protocol was approved by the Research Ethics Committee of Wenzhou Medical University (registration number L-2013-03). All authors have identified individual participants after data collection.

\section{Gensini score}

The severity of CAD was determined by Gensini scoring system which has been previously described [14]. Briefly, if any branches of main coronary artery Left Main Artery (LM), LAD, Left Circumflex Coronary Artery (LCX) and RCA has stenosis reaching 1-24\% of the internal lumen diameter, 1 point is given. Similarly, 2 is given for $25-49 \%$ stenosis, 4 for $50-74 \%$, 8 for $75-90 \%$, 16 for $91-99 \%$ and 32 for $100 \%$ or total occlusion. Depending on the lesion location, the single lesion score and the coefficient, the final Gensini total score was calculated.

\section{Human genomic DNA extraction}

A blood sample of $5 \mathrm{~mL}$ was collected into a tube containing ethylene diamine tetra acetic acid (EDTA) from the radial artery. After centrifugation, plasma was collected and stored at $-80{ }^{\circ} \mathrm{C}$ until use. Genomic DNA was extracted from cells by a DNA extraction kit (Tiangen Company, Beijing, China). The isolated DNA was also stored at $-80{ }^{\circ} \mathrm{C}$.

\section{MIF - 794 CATT5-8 genotyping}

Polymorphism was genotyped by sequencing of polymerase chain reaction (PCR) product as reported previously $[4,15]$. The forward primer was 5TTGCACCTATCAGAGACC-3 and the reverse primer was 5-TCCACTAATGGTAAACTCG-3. These primers were designed to amplify a 207 bp segment of the MIF promoter region. PCR was carried out in a volume of $25 \mu \mathrm{l}$. The reaction conditions of PCR were as follows: initial denaturation at $95{ }^{\circ} \mathrm{C}$ for $5 \mathrm{~min}$, followed by 35 cycles at $95{ }^{\circ} \mathrm{C}$ for $30 \mathrm{~s}, 60{ }^{\circ} \mathrm{C}$ for $30 \mathrm{~s}$, and $72{ }^{\circ} \mathrm{C}$ for $1 \mathrm{~min}$, with final extension at

Table 1 Clinical and biochemical characteristics by study group

\begin{tabular}{|c|c|c|c|c|}
\hline & $\begin{array}{l}\text { Control group } \\
(n=186)\end{array}$ & $\begin{array}{l}\text { CHD group } \\
(n=70)\end{array}$ & $F / x^{2}$ & $P$ value \\
\hline$\overline{M e a n}$ age(year) ${ }^{a}$ & $60.78 \pm 9.26$ & $66.71+10.25$ & 1.24 & 0.081 \\
\hline $\begin{array}{l}\text { Gander }^{\mathrm{b}} \\
\text { (male/female) }\end{array}$ & $84 / 102$ & $44 / 26$ & 6.37 & 0.012 \\
\hline cigarette smoking $(\%)^{b}$ & $31.6 \%$ & $33.3 \%$ & 0.236 & 0.627 \\
\hline Drinking(\%) ${ }^{\mathrm{b}}$ & $18.3 \%$ & $24.3 \%$ & 0.677 & 0.41 \\
\hline Hypertension $(\%)^{b, c}$ & $66.7 \%$ & $68.6 \%$ & 0.054 & 0.817 \\
\hline Hyperlipidemia $(\%)^{\mathrm{b}, \mathrm{d}}$ & $16.7 \%$ & $37.1 \%$ & 6.76 & 0.009 \\
\hline Diabetes $(\%)^{\mathrm{b}, \mathrm{e}}$ & $3.3 \%$ & $7.1 \%$ & 0.92 & 0.337 \\
\hline
\end{tabular}

${ }^{\mathrm{a}}$ Data presented as mean \pm SD. Student's t-test

${ }^{\mathrm{b}} \mathrm{Chi}$-square

cblood pressure $\geq 140 / 90 \mathrm{mmHg}$

${ }^{\mathrm{d} L D L-C} \geq 120 \mathrm{mg} / \mathrm{dl}$

${ }^{\mathrm{e}} \mathrm{FPG} \geq 7.0 \mathrm{mmol} / \mathrm{l}$ and/or OGTT $2 \mathrm{~h} \mathrm{FPG} \geq 11.1 \mathrm{mmol} / \mathrm{l}$ 
Table 2 Genotype and allele frequencies of -794 CATT5-8 MIF polymorphisms

\begin{tabular}{llllll}
\hline Polymorphisms & $\begin{array}{l}\text { Genotypes/ } \\
\text { alleles }\end{array}$ & $\begin{array}{l}\text { CHD group } \\
n=70\end{array}$ & $\begin{array}{l}\text { Control group } \\
n=186\end{array}$ & $x^{2}$ & $P$ value \\
\hline -794CATT & $5 / 5$ & $11(15.7)$ & $31(17.6)$ & & \\
$6 / 6$ & $16(22.9)$ & $30(17.0)$ & & \\
$7 / 7$ & $3(4.3)$ & $7(4.0)$ & & \\
$5 / 6$ & $22(31.4)$ & $56(31.8)$ & & \\
$5 / 7$ & $10(14.3)$ & $24(13.6)$ & & \\
$6 / 7$ & $8(11.4)$ & $26(14.8)$ & & \\
$6 / 8$ & $0(0)$ & $1(0.54)$ & 0.464 & 0.834 \\
5 & $54(38.6)$ & $132(35.5)$ & & \\
6 & $62(44.3)$ & $190(51.1)$ & & \\
7 & $24(17.1)$ & $49(13.2)$ & & \\
8 & $0(0)$ & $1(0.27)$ & 0.003 & 0.959
\end{tabular}

${ }^{\mathrm{a} C h i-s q u a r e ~ t e s t ~} x^{2}$

$72{ }^{\circ} \mathrm{C}$ for $10 \mathrm{~min}$. PCR products were revealed by agarose gel electrophoresis.

Genomic DNA was extracted from blood collected into tubes containing EDTA. The DNA of individuals previously sequenced was used as a template to generate control DNA fragments, using BigDye Terminator v1.1, in order to correlate the fragment size observed on the ABI 310 analyzer with the number of CATT repeats in the test samples [16]. (Sequenced by Shanghai Hybio BioTechnology Co., Ltd.).

\section{Analysis of serum MIF levels}

The plasma concentrations of MIF were measured using an enzyme linked immunosorbent assay (ELISA) kit according to the manufacturer's instructions (R\&D, USA).

\section{Statistic analyses}

MIF genotype and allele frequencies were analyzed using SPSS17.0 statistical software. The allele and genotype distributions were estimated by gene counting, and distribution of the polymorphic variants was tested against Hardy-Weinberg $(\mathrm{H}-\mathrm{W})$ equilibrium by $\mathrm{X}^{2}$ analysis. Plasma MIF concentrations were expressed as means \pm SD. For comparisons between two groups, we determined the significance of differences between means by t-tests. Comparisons between multiple groups were performed by ANOVA. $P \leq 0.05$ was considered statistically significant.

\section{Results}

Frequencies of MIF - 794 alleles and genotypes of CAD patients and controls

There were no significant differences in age, cigarette smoking, drinking hypertension, and diabetes except gender, $(P>0.05)$ (Table 1$)$ between the CAD patients and the control. Both CAD patients and controls were in Hardy-Weinberg equilibrium with MIF -794CATT5-8 genotypes' distribution $(P>0.05)$. There were seven kinds of genotypes and four kinds of alleles in these two groups (Table 2). The comparative analysis of genotype and allele frequencies of -794 CATT5-8 polymorphisms between groups did not show significant differences $(P>0.05)$.

\section{The plasma concentration of MIF}

The plasma MIF concentration of CAD group was $65.75 \pm 6.32 \mu \mathrm{g} / \mathrm{L}$, significantly higher than that of nonCAD group $(51.13 \pm 7.33 \mu \mathrm{g} / \mathrm{L}, P<0.05)$, as we known before [12]. MIF serum levels were similar among CATT (5), CATT (6), and CATT (7) allele carriers. (Table 3).

In CAD patients the plasma MIF concentration of the carriers of CATT(5) allele was significantly lower than that of the CATT(7) allele carriers $(P<0.05)$ (Fig. 1a). When MIF serum levels were compared among CAD patients with different genotypes, we did not observe significant difference. In the CAD patients, the plasma MIF concentration was lower in the CATT(5/5) group than CATT(6/6) and CATT(7/7) groups but the difference was not statically significant $(P>0.05)$ (Fig. 1b). While in normalcoronary subjects, we did not observe a correlation between MIF serum levels with allele and genotypes $(P>0.05)$ (data not shown).

Table 3 The plasma MIF concentration

\begin{tabular}{llll}
\hline & CHD group $(n=70)$ & Control group $(n=186)$ & $P^{*}$ value \\
mean concentration of MIF + SD & $65.75 \pm 6.32$ & $51.13 \pm 7.33$ & \\
median of MIF & 65.66 & 50.01 & 0.00 \\
& alleles $5(2 n=186)$ & alleles $6(2 n=252)$ & alleles $7(2 n=73)$ \\
mean concentration of MIF + SD & $58.68+7.80$ & $60.22+9.77$ & $62.09+5.32$ \\
median of MIF & $58.275+1.42$ & $59.75+0.98$ & $61.79+0.51$ \\
\hline
\end{tabular}

*Student's t-test

${ }^{\#}$ Chi-square test $x^{2}$ 

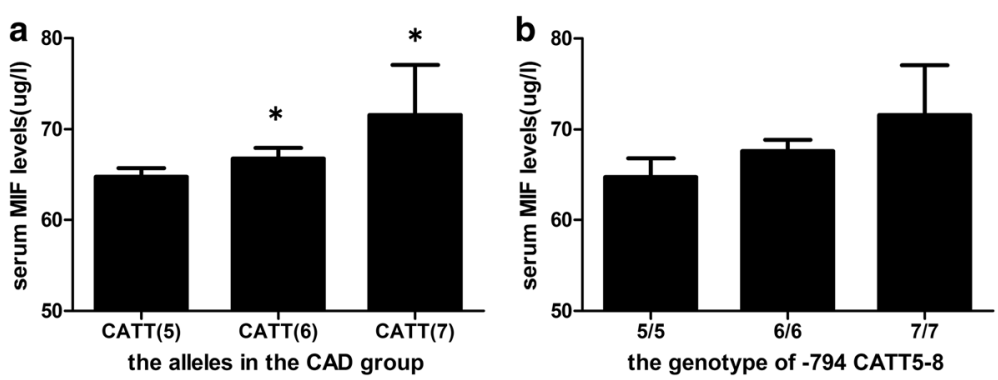

Fig. 1 the plasma MIF concentration of carriers of MIF -794CATT5-8 alleles and genotypes in CAD patients. a The plasma MIF concentration of the carriers of CATT(5) allele was significantly lower than that of the CATT(7) allele carriers $(P<0.05)$. $\mathbf{b}$ The plasma MIF concentration was lower in the CATT(5/5) group than CATT(6/6) and CATT(7/7) groups but the difference was not statically significant $(P>0.05)$

Frequencies of MIF- CATT $_{5-8}$ alleles among CAD patients Patients were classified into three subgroups according to the Gensini system. There was no difference in MIFCATT5-8 allele frequency in CAD patients with different scores (Table 4).

\section{Discussion}

Despite the improvements in medical treatments and subsequent survival rates, CAD is still the leading cause of death worldwide [1]. In addition, it is well documented that there is strong relationship between many genetic variants and environmental factor in CAD. Therefore, the knowledge of genetic mechanisms of CAD is helpful to develop new disease prevention and treatment strategies.

Polymorphism of MIF on the -173 position has been reported in several inflammatory diseases, including ulcerative colitis (UC) [10], psoriasis [9] and tuberculosis (TB) [11]. Several studies have reported the association between MIF794CATT gene polymorphism, MIF protein level and CAD. For example in Western Mexico, MIF794CATT (6/ 7) genotype was found to correlate with the onset of acute coronary syndrome [17]. Lan et al. reported that discovered polymorphism of the MIF gene was associated with the severity of carotid atherosclerotic plaque [18]. In the present study, MIF794CATT gene polymorphism and CAD incidence were not significantly correlated, but in

Table 4 the relationship between CHD class and the allele frequency of MIF-794 CATT $5-8$

\begin{tabular}{lll}
\hline $\begin{array}{l}\text { CHD class } \\
\text { (Gensini's intergral degree) }\end{array}$ & \multicolumn{2}{l}{$\begin{array}{l}\text { the allele frequency of } \\
\text { MIF-794 CATT }\end{array}$} \\
\cline { 2 - 3 } & 5 & 6 \\
\hline$\leqq 20$ & 16 & 10 \\
$20-40$ & 14 & 10 \\
$\geqq 40$ & 13 & 4 \\
$X^{2}$ & 1.55 & \\
$P^{a}$ value & 0.46 & \\
\hline
\end{tabular}

${ }^{\mathrm{a} C h i-s q u a r e ~ t e s t}$ the CAD patients, CATT (5) allele carriers had lower serum MIF concentration than the other two groups. Indeed, in vitro studies found that, CATT (5) allele has the lowest level of basic and stimulated MIF promoter activity when compared to the CATT (6) and CATT (7) alleles $[16,19]$. However, it is still unknown which transcription factors regulate the expression of MIF by binding to CATT gene promoter region [20].

We found that serum MIF concentrations were significantly higher in CAD patients than the non-CAD patients, which is inconsistence with the previous finding that increased incidence MIF protein concentration is related to increased incidence of CAD [12]. However, MIF794CATT5-8 allele were not associated with the severity of CAD.

Our study is based at single center and the cases are limited because of time and geographical restrictions. Further studies with bigger sample size and patients from more cities are needed to confirm this primary conclusion.

\section{Conclusion}

To sum up, there is no significant correlation between the polymorphism of 794CATT gene and the severity of CAD.

\section{Abbreviations}

CAD: Coronary Atherosclerotic Disease; CAG: Coronary Angiography;

EDTA: Ethylene Diamine Tetra Acetic Acid; ELISA: Enzyme Linked Immunosorbent Assay; LAD: Left Anterior Descending; LCX: Left Circumflex Coronary Artery; LM: Left Main Artery; MIF: Migration Inhibitory Factor; PCR: Polymerase Chain Reaction; RCA: Right Coronary Artery; SMCs: Vascular smooth muscle cells; SNPs: Single Nucleotide Polymorphism;

TB: Tuberculosis; UC: Ulcerative Colitis

\section{Acknowledgements}

Not applicable.

\section{Availability of data and materials}

The datasets used and/or analysed during the current study available from the corresponding author on reasonable request.

Funding

The authors disclosed receipt of the following financial support for the research and authorship of this article: National Natural Science Foundation of China [Nos. 81,573,185], Natural Science Foundation of Zhejiang Province 
[Nos. 2014C33163], Wenzhou Municipal Science and Technology Bureau [Nos. H20140001, Nos.Y20130167].

\section{Authors' contributions}

LQ: research design, perform experiments, collect data and analysis of data, draft manuscript. XW: perform experiments, collect data and analysis of data. ST: perform experiments, draft manuscript. LT: perform experiments, collect data and analysis of data. SW: perform experiments, collect data and analysis of data. GL: collect data, draft manuscript. LW: perform experiments, collect data and analysis of data. JW1(Jiao-Ni Wang): perform experiments, collect data and analysis of data. JW2(Jie Wang): collect data and analysis of data. JL: research design, revising manuscript. JT: research design, draft manuscript. KJ: research design, draft manuscript. All authors read and approved the final manuscript.

\section{Competing interests}

The authors declare that there is no conflict of interests regarding the publication of this paper.

\section{Consent for publication}

Not applicable.

\section{Ethics approval and consent to participate}

This protocol was approved by the Research Ethics Committee of Wenzhou Medical University (registration number L-2013-03).The informed consent was obtained from all participants.

\section{Publisher's Note}

Springer Nature remains neutral with regard to jurisdictional claims in published maps and institutional affiliations.

\section{Author details}

'Department of Cardiology, the Second Affiliated Hospital, Wenzhou Medical University, Wenzhou, Zhejiang 325000, China. 'epartment of Cardiology, Yiwu Central Hospital, Yiwu 322000, China.

Received: 25 October 2016 Accepted: 15 May 2017

Published online: 02 June 2017

\section{References}

1. Zaiying Lu NZ, Xie Y, Hu P. Internal medicine: People's medical publishing house; 2008. p. 274.

2. Rifai N, Ridker PM. Inflammatory markers and coronary heart disease. Curr Opin Lipidol. 2002:13(4):383-9.

3. Pamukcu B, Lip GY, Devitt A, et al. The role of monocytes in atherosclerotic coronary artery disease. Ann Med. 2010:42(6):394-403.

4. Leng L, Bucala R. Insight into the biology of macrophage migration inhibitory factor (MIF) revealed by the cloning of its cell surface receptor. Cell Res. 2006;16(2):162-8.

5. Bernhagen J, Calandra T, Mitchell RA, et al. MIF is a pituitary-derived cytokine that potentiates lethal endotoxaemia. Nature. 1993;365(6448):756-9.

6. Calandra T, Bernhagen J, Mitchell RA, et al. The macrophage is an important and previously unrecognized source of macrophage migration inhibitory factor. J Exp Med. 1994;179(6):1895-902

7. Burger-Kentischer A, Goebel H, Seiler R, et al. Expression of macrophage migration inhibitory factor in different stages of human atherosclerosis. Circulation. 2002:105(13):1561-6.

8. Willis MS, Carlson DL, Dimaio JM, et al. Macrophage migration inhibitory factor mediates late cardiac dysfunction after burn injury. Am J Phys Heart Circ Phys. 2005;288(2):H795-804.

9. Wu J, Chen F, Zhang X, et al. Association of MIF promoter polymorphisms with psoriasis in a Han population in northeastern China. J Dermatol Sci. 2009;53(3):212-5

10. Shiroeda H, Tahara T, Nakamura M, et al. Association between functional promoter polymorphisms of macrophage migration inhibitory factor (MIF) gene and ulcerative colitis in Japan. Cytokine. 2010:51(2):173-7.

11. Li Y, Zeng Z, Deng S. Study of the relationship between human MIF level, MIF-794CATT5-8 microsatellite polymorphism, and susceptibility of tuberculosis in Southwest China. Braz J Infect Dis. 2012;16(4):383-6.
12. Ji K, Wang $X$, Li J, et al. Macrophage migration inhibitory factor polymorphism is associated with susceptibility to inflammatory coronary heart disease. Biomed Res Int. 2015;2015:315174.

13. Barton A, Lamb R, Symmons $D$, et al. Macrophage migration inhibitory factor (MIF) gene polymorphism is associated with susceptibility to but not severity of inflammatory polyarthritis. Genes Immun. 2003:4(7):487-91.

14. Gensini GG. A more meaningful scoring system for determining the severity of coronary heart disease. Am J Cardiol. 1983;51(3):606.

15. Eriksson EE. Mechanisms of leukocyte recruitment to atherosclerotic lesions: future prospects. Curr Opin Lipidol. 2004;15(5):553-8.

16. Baugh JA, Chitnis S, Donnelly SC, et al. A functional promoter polymorphism in the macrophage migration inhibitory factor (MIF) gene associated with disease severity in rheumatoid arthritis. Genes Immun. 2002;3(3):170-6

17. Valdes-Alvarado E, Munoz-Valle JF, Valle $Y$, et al. Association between the -794 (CATT)5-8 MIF gene polymorphism and susceptibility to acute coronary syndrome in a western Mexican population; 2014. p. 704854.

18. Lan MY, Chang YY, Chen WH, et al. Association between MIF gene polymorphisms and carotid artery atherosclerosis. Biochem Biophys Res Commun. 2013:435(2):319-22.

19. Renner $\mathrm{P}$, Roger $\mathrm{T}, \mathrm{Bochud} \mathrm{PY}$, et al. A functional microsatellite of the macrophage migration inhibitory factor gene associated with meningococcal disease. FASEB J. 2012;26(2):907-16.

20. Radstake TR, Sweep FC, Welsing P, et al. Correlation of rheumatoid arthritis severity with the genetic functional variants and circulating levels of macrophage migration inhibitory factor. Arthritis Rheum. 2005;52(10):3020-9.

\section{Submit your next manuscript to BioMed Central and we will help you at every step:}

- We accept pre-submission inquiries

- Our selector tool helps you to find the most relevant journal

- We provide round the clock customer support

- Convenient online submission

- Thorough peer review

- Inclusion in PubMed and all major indexing services

- Maximum visibility for your research

Submit your manuscript at www.biomedcentral.com/submit
Biomed Central 